\title{
A step toward essential tremor gene discovery: identification of extreme phenotype and screening of HTRA2 and ANO3
}

Mathilde Renaud ${ }^{1,2,3}$, Christophe Marcel ${ }^{1,2}$, Gabrielle Rudolf 1,2,3, Mickaël Schaeffer ${ }^{2,4}$, Ouhaïd Lagha-Boukbiza ${ }^{1,2}$, Jean-Baptiste Chanson 1,2, Jamel Chelly ${ }^{2,3,5}$, Mathieu Anheim ${ }^{1,2,3}$ and Christine Tranchant ${ }^{1,2,3^{*}}$

\begin{abstract}
Background: Essential tremor (ET) is characterized by a frequent family history. No monogenic form of ET has been identified. We aimed at exploring ET patients to identify distinct subgroups and facilitate the identification of ET genes. We tested for the presence of HTRA2 p.G399S, and ANO3 p. W490C, p. R484 W and p. S685G mutations.

Methods: Between June 2011 and November 2013, all consecutive patients suspected with ET were prospectively included in a prospective, monocentric study. Family history, age at onset (AAO), features of tremor, benefit of alcohol and drugs, electrophysiological recording findings were collected. Sanger sequencing was performed for HTRA2 and ANO3 mutations screening.

Results: Sixty eight patients were investigated. Fourteen diagnosed with psychogenic (5) or dystonic tremor (9) were excluded. Regarding the 54 ET patients, mean AAO was 48 years (6-77), and mean disease duration 15 years (1-55). Bimodal distribution of AAO was consistent with phenotypic subgroups. In patients with AAO before 30 years, marked benefit of alcohol $(p<0.01)$ and ET family history $(p<0.01)$ were more frequent and the disease progression less severe $(p<0.0001)$. Neither HTRA2 nor ANO3 mutation were identified in our patients.

Conclusions: Our data support that distinct ET phenotypic subgroups may be encountered. We recommend to study separately extreme phenotypes of ET, particularly autosomal dominant families with early AAO ( $<30$ years) and marked benefit of alcohol, to facilitate the identification of ET genes. Electromyographic recording remains a support to distinguish ET from differential diagnosis. HTRA2 and ANO3 mutations are not common causes of ET.
\end{abstract}

Keywords: Essential tremor, Electrophysiological recording, Extreme phenotype, ANO3, HTRA2

\section{Background}

According to the Consensus Statement of the Movement Disorder Society (CSMDS) [1], essential tremor (ET) is characterized by: (1) a bilateral, largely symmetric, postural tremor with or without kinetic component, which affects hands and forearms, that is present for at least 5 years, visible and persistent, (2) additional or isolated tremor of the head may occur but in the absence of

\footnotetext{
*Correspondence: christine.tranchant@chru-strasbourg.fr

'Service of Neurology, University Hospital of Strasbourg, Hospital of Hautepierre, 1 avenue Molière, 67098 Strasbourg Cedex, France

${ }^{2}$ Fédération de Médecine Translationnelle de Strasbourg (FMTS), Université

de Strasbourg, Strasbourg, France

Full list of author information is available at the end of the article
}

abnormal posturing, and (3) exclusion of other causes for tremor (other neurological diseases, iatrogenic tremor, exaggerated physiological tremor, recent history of head trauma, psychogenic trigger). Nevertheless, debates continue about the definition of ET because it is an heterogeneous disorder that has no clear diagnostic test [2].

Clinical practice emphasizes ET heterogeneity, and the limits of the above definition: family history is frequent but may be lacking, histogram of age at onset (AAO) demonstrates bimodal distribution with a first peak in childhood or adolescence [3] and a second around the 5th and 6th decades. In the same way, unilateral cases 
have been described [4], or cases associated with other motor (rest tremor, cerebellar ataxia) or non-motor (depressive syndrome, sleep disorders, cognitive decline) signs have been reported [5], and response to alcohol or to beta-blockers are only optional. Moreover, distinct pathological patterns have been reported [6]: one with cerebellar lesions, loss of Purkinje cells and presence of torpedoes; another with brainstem Lewy bodies.

Such heterogeneity added to the lack of stringent diagnostic criteria or of biomarkers and to the evidence for non mendelian inheritance could explain the weak profitability of genetic studies and their difficulty to find reproducible data [7]. Indeed, a mutation in the FUS gene [8] was found in only one ET family. LINGO1 and SLC1A2 polymorphisms have been only identified in genome-wide association studies [9-11]. An HTRA2 variant has very recently been associated in one family with ET and optional parkinsonism [12]. However, a clear, monogenic form of ET is still lacking. ANO3 mutations were reported in a spectrum of dystonic patients initially presumed to have familial essential tremor [13].

We aimed at analysing a cohort of patients with ET to identify distinct phenotypic subgroups in order to facilitate the identification of genes responsible for ET, and searched for HTRA2 and ANO3 mutations recently identified as causes of family ET with extrapyramidal features.

\section{Methods}

From June 2011 to September 2013, all the patients referred in our tertiary movement disorders center with suspected ET, according to the CSMDS [1] definition were included in a prospective, monocentric study. All patients provided informed consent for the study and to be videotaped, and the local ethics committee approved the study.

\section{Clinical examination}

Following items were collected: family history, AAO, current age, clinical features of tremor, severity scales (The Essential Tremor Rating Assessment Scale score (TETRAS) performance subscale [14]), marked benefit of alcohol (patients were asked to answer to the question "does your tremor markedly improve following alcohol consumption") and to drugs, clinical signs associated to tremor, severity of disease progression (defined by the TETRAS performance subscale/duration of ET ratio). A video recording was performed for each patient, enabling the confirmation of the diagnosis of ET by a second neurologist devoted to movement disorders.

\section{Electrophysiological recordings}

Using (1) surface electrodes on extensor and flexor muscles of both forearms, and on cervical muscles, and (2) an unidirectional piezoelectric accelerometer placed on the index finger of dominant hand, the following items were analysed: the conditions of occurrence of the tremor (rest, posture, intention), frequency, regularity, agonist-antagonist synchronicity, distribution, and distractibility. Accelerometer signal was bandpass filtered at $20-500 \mathrm{~Hz}$. Postural and intention tremor were analyzed during postural maintenance and action (goal-directed movements and drawing). The effect of mental calculation was tested at rest. Patients with atypical tremor including distractible electrophysiological patterns (variations of frequency, transient disappearance of the tremor) suggestive of psychogenic tremor and patients with suspected dystonic tremor (abnormal posture, agonist-antagonist muscles co-contraction) were excluded from the study.

\section{Biology and Sanger sequencing}

Cupper, ceruloplasmin and thyroid stimulating hormone (TSH) serum levels were analysed in each patient. FMR1 premutation was investigated in all patients with onset after 50 years in order to rule out fragile X-associated tremor ataxia syndrome (FXTAS) [15]. For screening of HTRA2 p.G399S, and ANO3 p.W490C, p.R484 W and p.S685G variants recently reported as causes of ET, DNA was extracted from whole blood using illustra ${ }^{\mathrm{am}}$ DNA Extraction kit BACC3 (GE Healthcare UK).. The coding exons of HTRA2 and ANO3, reported recently $[12,13]$ were screened for mutations by Sanger sequencing of genomic DNA. Fifty-microliter PCR reactions were carried out with $40 \mathrm{ng}$ of genomic DNA and 10pmol of each pair of primer of forward and reverse primers using KAPA Hifi Hotstart ReadyMix PCR Kit (KAPABiosystems, Boston, Masschusetts, United States). Primer pairs are available upon request. All PCR products were purified and both strands were sequenced at GATC Biotech (http//www.gatc-biotech.com/). Sequenced were analysed using the SEQSCAPE v2.5 software (Applied Biosystems, Courtaboeuf, France).

\section{Brain MRI and DAT-SPECT}

Cerebral MRI was performed in 28 cases and, dopamine transporter single-photon emission tomography (DATSPECT) was performed in 8 of the 10 patients who also had rest tremor.

\section{Statistical analysis}

Statistical analyses were performed using parametric and non-parametric tests, according to the data distributions. Association between categorical variables was assessed using Fisher-exact tests, because of the small populations in each level of the qualitative variables.

Correlation between quantitative variables has been tested using Pearson correlation tests and Spearman correlation tests, in order to check for linear and non-linear correlation assumption. 
Link between a qualitative and a quantitative variable was checked using the non-parametric Kruskal-Wallis test, because of the nature of continuous variables distributions. The Gaussian assumption of these formers was not always checked (Shapiro-Wilk test), implying the use of those non-parametric tests.

Alpha risk was fixed to 5\%. Analyses were performed using the R software (version 3.0.2).

\section{Results}

Sixty-eight patients were investigated. Taken together, clinical and neurophysiological findings were finally consistent with psychogenic tremor in 5 patients and dystonic tremor in 9 patients who were excluded.

The main clinical and electrophysiological data of the remaining 54 patients are summarized in Tables 1 and 2 and in Fig. 1.

\section{Patients}

The 54 studied patients had a mean age of 62 years (range, 18 to 80 ) and had a mean history of tremor of 14.5 years (range, 1 to 55 ). The mean AAO was 48 years (range, 6 to 77). Tremor age of onset showed a bimodal distribution (Fig. 1). A positive family history of tremor was present in 29 (54\%) patients. The mean TETRAS performance subscale was 8.5 (range, 1 to 21). Marked benefit of alcohol was found in 13/44 (30\%) patients. Marked benefit of alcohol as well as positive family history of ET were correlated with earlier age of onset $(p=0.008$ and $p=0.0007$, respectively). TETRAS score was higher when head tremor was present $(p<0.001)$ but head tremor was positively correlated with neither age of onset nor tremor duration.

\section{Tremor anatomic distribution and tremor patterns}

All patients had bilateral upper extremities tremor, 4 (7\%) an associated lower limbs and 14 (26\%) an associated head tremor. Mean frequency of tremor was $6.3 \mathrm{~Hz}$ (3.8-11) in upper extremities. All patients had postural tremor, 46 patients (85\%) an associated intentional tremor and $10(18 \%)$ an associated rest tremor.

\section{Biological, brain MRI, DAT-SPECT and genetic data}

Biological analysis (cupper, ceruloplasmin, TSH) was normal in all patients. All the 18 patients investigated for FMR1 permutation were negative. No patients had HTRA2 p.G399S, or ANO3 p.W490C, p.R484 W and p.S685G variant.

Brain MRI was normal in 13/28 cases, but demonstrated vascular leucopathy in 9, cortical frontal atrophy in 2, incidental meningioma in 2 (a parieto-occipital parasagittal left meningomia $(6 \mathrm{~cm}$ in diameter $)$ and a frontal right meningomia (2 $\mathrm{cm}$ in diameter)), and asymptomatic aneurysm of a cerebral artery in 2 patients.
Table 1 Demographic, medical and upper limb electrophysiologic data of the cohort

\begin{tabular}{|c|c|}
\hline & Patients $(n=54)$ \\
\hline \multicolumn{2}{|l|}{ Demographic features } \\
\hline Female & $25(46 \%)$ \\
\hline Male & $29(54 \%)$ \\
\hline Mean age at evaluation, range & 62 years $(18-80)$ \\
\hline Mean AAO of tremor, range & 48 years $(6-77)$ \\
\hline Duration of tremor, range & 14.5 years $(1-55)$ \\
\hline Family history of essential tremor & $29(54 \%)$ \\
\hline Family history of Parkinson's disease & $2(3.5 \%)$ \\
\hline Family history of ataxia & 0 \\
\hline Family history of other neurodegenerative disease & $2(3.5 \%)$ \\
\hline Marked benefit of alcohol ( $n=44$ patients) & $13(30 \%)$ \\
\hline Increased at morning ${ }^{a}$ & $10(20 \%)$ \\
\hline Increased with emotions ${ }^{\mathrm{b}}$ & $44(82 \%)$ \\
\hline Increased with physical exercise ${ }^{c}$ & $22(41 \%)$ \\
\hline \multicolumn{2}{|l|}{ Initial location of tremor } \\
\hline One hand & $27(50 \%)$ \\
\hline Dominant hand & $23(42 \%)$ \\
\hline Two hands & $27(50 \%)$ \\
\hline \multicolumn{2}{|l|}{ Current location of tremor } \\
\hline Upper limbs & $54(100 \%)$ \\
\hline Bilateral & $54(100 \%)$ \\
\hline Unilateral & 0 \\
\hline Symmetrical & $17(32 \%)$ \\
\hline Lower limbs & $4(7 \%)$ \\
\hline Head & $14(26 \%)$ \\
\hline Voice & $9(17 \%)$ \\
\hline Trunk & $2(4 \%)$ \\
\hline \multicolumn{2}{|l|}{ Other clinical signs } \\
\hline Akinesia & $5(9 \%)$ \\
\hline Ataxia & $2(4 \%)$ \\
\hline TETRAS score (performance subscale), range & $8.5(1-21)$ \\
\hline \multicolumn{2}{|l|}{ Electrophysiologic findings in upper limb extremities } \\
\hline Rest tremor & $10(18 \%)$ \\
\hline Postural tremor & $54(100 \%)$ \\
\hline Intentional tremor & $46(85 \%)$ \\
\hline Postural tremor only & $5(9 \%)$ \\
\hline Rest, postural and intentional tremor & $8(15 \%)$ \\
\hline Frequency of postural tremor & $6.3 \mathrm{~Hz}(3.8-11)$ \\
\hline
\end{tabular}

TETRAS The Essential Tremor Rating Assessment Scale; AAO age at onset, $\mathrm{Hz}$ Hertz

a amplitude of tremor more important relative to the rest of the day

bamplitude of tremor more important with emotions (happiness, sadness)

camplitude of tremor more important with sustained exercise 
Table 2 Correlation between age at onset and other characteristics of tremor ( $n=42$ patients)

\begin{tabular}{|c|c|c|c|}
\hline & $\begin{array}{l}\text { AAO } \leq 30 \text { years (early onset ET) } \\
(n=14)\end{array}$ & $\begin{array}{l}\text { AAO } \geq 55 \text { years (late onset ET) } \\
(n=28)\end{array}$ & $p$ \\
\hline Family history of essential tremor $(n=42)$ & $93 \%(n=13)$ & $36 \%(n=10)$ & 0.0007 \\
\hline Benefit of alcohol $(n=33)$ & $54 \%(n=6)$ & $9 \%(n=2)$ & 0.008 \\
\hline Duration of tremor, years (SD) $(n=42)$ & $29(+/-16)$ & $6.5(+/-6)$ & $<0.0001$ \\
\hline TETRAS score/duration of tremor (SD) $(n=42)$ & $0.4(+/-0.2)$ & $2.3(+/-2.3)$ & $<0.0001$ \\
\hline Head tremor $(n=42)$ & $21 \%(n=3)$ & $29 \%(n=8)$ & 1.00 \\
\hline Lower limbs tremor $(n=42)$ & $21 \%(n=3)$ & $0 \%(n=0)$ & 0.03 \\
\hline Rest tremor $(n=42)$ & $21.4 \%(n=3)$ & $17.8 \%(n=5)$ & 0.85 \\
\hline Mean frequency, Hertz (SD) $(n=42)$ & $7.0(+/-2)$ & $6.2(+/-1)$ & 0.23 \\
\hline TETRAS score (SD) $(n=42)$ & $9.6(+/-4)$ & $7.8(+/-5)$ & 0.15 \\
\hline Response to beta-blockers $(n=23)$ & $77 \%(n=10)$ & $70 \%(n=7)$ & 1.00 \\
\hline Response to primidone $(n=10)$ & $80 \%(n=4)$ & $60 \%(n=3)$ & 1.00 \\
\hline
\end{tabular}

$A A O$ age at onset, SD standard deviation, TETRAS The Essential Tremor Rating Assessment Scale

DAT-SPECT demonstrated the loss of dopaminergic neurons in $4 / 8$ patients with rest tremor.

\section{Medications}

Treatments and efficacy of treatments are summarized in Table 3. None of the patients had received antipsychotic or anti-emetic drugs. None of the following factors were found predictive of response to main antitremor drugs (propranolol, primidone, topiramate): ET family history $(p=0.28, p=1.0, p=1.0$, respectively); marked benefit of alcohol $(p=1.0, p=1.0, p=1.0$, respectively); associated rest tremor $(p=0.32, p=1.0, p=1.0$, respectively); symmetrical tremor $(p=0.62, p=01.0, p=1.0$, respectively); associated head tremor $(p=1.0, p=1.0$, $p=1.0$, respectively); associated lower limbs tremor $(p=1.0, p=0.30, p=1.0$, respectively); frequency $(p=0.67$, $p=0.73, p=0.65$, respectively); TETRAS performance subscale $(p=0.97, p=0.35, p=0.65$, respectively); evolution speed (ratio TETRAS performance subscale/duration of tremor) ( $p=0.92, p=0.21, p=0.65$, respectively).

\section{Discussion}

We aimed at identifying extreme phenotype of ET that could be distinguished from other ET variants in order to find genes responsible for ET which appears to be heterogeneous regarding both clinical and genetic basis. Indeed, the identification of gene causing ET remains an important genetic challenge in the field of movement disorders.

Our study confirmed the bimodal distribution of AAO and the frequent positive family history of ET $[16,17]$ that support genetic heterogeneity of ET. In the same way, our results further strengthen previous data showing

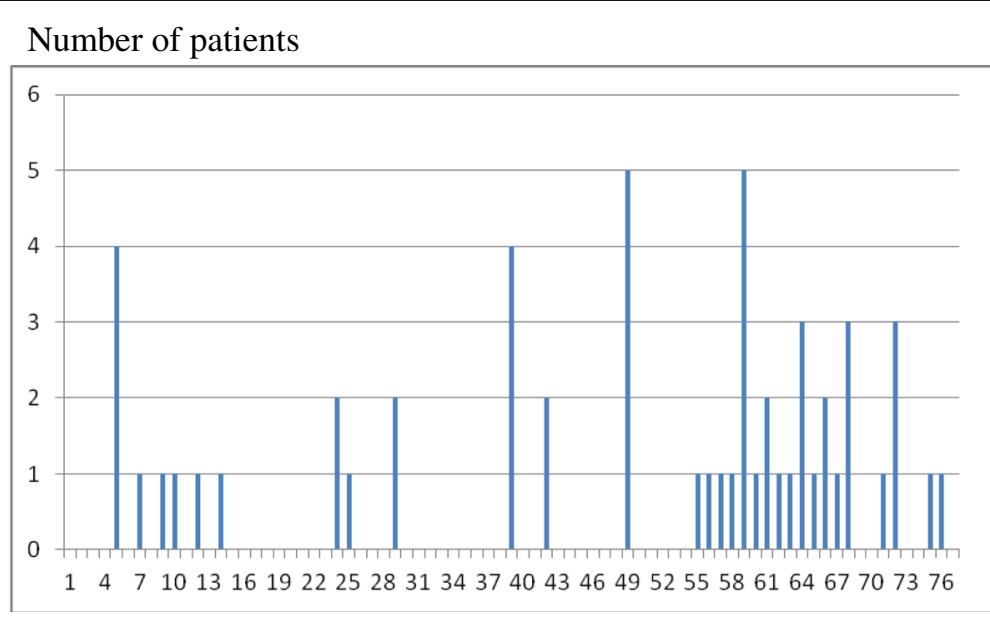

Age at onset

Fig. 1 Bimodal distribution of age at onset of tremor 
Table 3 Treatments and efficacy of treatments

\begin{tabular}{ll}
\hline Medications & Patients $(n=54$ patients) \\
\hline Propranolol ( $n=31$ patients) & $24(77 \%)$ \\
Positive effect of propranolol & $109(40-300)$ \\
Mean posology, en milligram/day, range & $57(1-360)$ \\
Mean length of treatment, months, range & \\
Primidone ( $n=16$ patients) & $13(81 \%)$ \\
Positive effect of primidone & $338(10-750)$ \\
Mean posology, en milligram/day, range & $15(1-60)$ \\
Mean length of treatment, months, range & \\
Topiramate ( $n=7$ patients) & $3(43 \%)$ \\
Positive effect of topiramate & $75(25-150)$ \\
Mean posology, en milligram/day, range & $11(1-36)$ \\
Mean length of treatment, months, range & $2(4 \%)$ \\
Chronic high frequency thalamic stimulation &
\end{tabular}

that there is a correlation between a family history of tremor and a younger AAO [18].

We found that early onset of tremor, marked benefit of alcohol consumption and positive family history of ET should be considered as such peculiar phenotype also characterized by a less severe progression of the disease. Thus, in order to identify genes causing ET, we recommend to perform genetic analysis in patients with such extreme phenotype. However, these new findings need to be confirmed in larger series. Benefit of alcohol could be quantitatively assessed [19] and could be underpinned by a peculiar pathophysiological process. A family history is clearly suggestive of inheritance of the disease and in the same way, the youngest the onset, the most probable the inheritance.

For the identification of such peculiar phenotype, electrophysiological recording remains a strong support. Indeed, we confirmed the difficulty in some patients to distinguish ET from differential diagnosis such as dystonic tremor or to some extent psychogenic tremor. Therefore, we also recommend to perform electrophysiological recordings for the selection of patients with genuine ET for future genetic studies.

We found that tremor frequency and head tremor should not be considered as determinant factors for the identification of a peculiar phenotype. In our series, some findings could be explained by the natural history of ET rather than by different phenotypes of ET, since patients with earlier age at onset had longer disease duration. Indeed, an age-associated decrease in tremor frequency (approximately 0.06 to $0.08 \mathrm{~Hz} /$ year [20]) has been confirmed in our series, where tremor frequency was negatively correlated with current age $(p<0.01)$ and TETRAS subscale performance $(p<0.01)$. Likewise, head tremor which was present in only 11 patients $(26.2 \%$, against 31 to $59.4 \%$ in previous series) [3, 21], was only correlated with tremor severity $(p<0.001)$. In the same way, the higher frequency of lower limb tremor in patients with earlier age at onset could be due to the natural history of the disease rather than to a peculiar phenotype. However, this statement remains to be confirmed by further studies.

Rest tremor was present in $18 \%$ patients, but in opposite to previous data [22], no correlation was found with neither tremor duration $(p=0.31)$ nor TETRAS subscale performance $(p=0.2)$. Some authors suggested that in patients with severe, old and disseminated ET, rest tremor could be explained by diffusion of the pathologic process to the dopaminergic system. However recent post-mortem studies of a few ET patients with rest tremor did not reveal Lewy body pathology [6], and in our series, only four from 8 patients with rest tremor and who underwent DAT scan, demonstrated dopaminergic denervation.

Nevertheless, epidemiological studies have shown that ET could be a risk factor for Parkinson's disease (PD) [23]. Additional non motor signs such as anosmia or rapid eye movement behaviour disorder in previous series [5] argue for a link between PD and ET. Whether patients affected with both ET and PD should be considered as suitable for the identification of tremor-causing genes needs to be confirmed in a near future. HTRA2 p.G399S variant has recently been identified to be a risk factor with overlapping family ET and PD but we did not find this variant in our cohort [12]. In the same way, this variant has been very recently searched for in a large series of ET and was finally considered as not a common cause of ET [24, 25]. We did not find ANO3 mutations in any of our patient using Sanger sequencing. These data have to be replicated in a larger sample of ET patients.

Finally, our series confirmed the therapeutic efficacy of propranolol and primidone [26], and to lesser extent of topiramate, but did not identify any predictors of positive response for any of these drugs. Main limitation of our study is the relatively small number of patients, compared to previous series $[3,16,21]$. However its originality consists of the theory of extreme phenotypes, of comprehensive clinical and electrophysiological data, and of up-to-date genetic studies.

\section{Conclusions}

Larger cohorts are probably needed to confirm that the factors identified in the present study (early age of onset, marked benefit of alcohol, dominantly inherited family history of ET) correspond to an extreme phenotype of ET. Our results suggest to study separately patients with such peculiar phenotype in order to finally find gene responsible for ET. 


\section{Abbreviations}

AAO: Age at onset; DAT-SPECT: Dopamine transporter single-photon emission tomography; ET: Essential tremor; FXTAS: Fragile X-associated tremor ataxia syndrome; Hz: Hertz; PD: Parkinson's disease; TETRAS: The essential tremor rating assessment scale score; TSH: Thyroid stimulating hormone

\section{Funding}

This study was supported by funds from APTES (association des personnes concernées par le tremblement essentiel).

\section{Availability of data and materials}

Data and materials are available in the movement disorders center, department of neurology, Hôpitaux Universitaires de Strasbourg, Hôpital de Hautepierre, 67098 Strasbourg Cedex, France, only by doctors of our service.

\section{Authors' contributions}

MR acquisition and analysis of data, conception, writing of the first draft, review and critique of the manuscript. CM acquisition and analysis of clinical and electrophysiological data, review and critique of the manuscript. GR acquisition and analysis of genetic data, review and critique of the manuscript. MS design and execution of statistical data, review and critique of the manuscript. OLB acquisition and analysis of clinical and electrophysiological data, review and critique of the manuscript. JBC acquisition and analysis of clinical and electrophysiological data, review and critique of the manuscript. JC analysis of genetic data, review and critique of the manuscript. MA organization and execution of the research project, review and critique of the manuscript. CT conception, organization and execution of the research project, analysis of data, review and critique of the manuscript. All authors read and approved the final manuscript.

\section{Competing interests}

The authors declare that they have no competing interests.

\section{Consent for publication}

The final manuscript has been seen and approved by all authors, and all authors accepted full responsibility for the design and conduct of the article, had access to the data, controlled the decision to publish.

\section{Ethics approval and consent to participate}

All patients provided informed consent for the study and to be videotaped, and the local ethics committee of the university hospital of Strasbourg approved the study.

\section{Author details}

'Service of Neurology, University Hospital of Strasbourg, Hospital of Hautepierre, 1 avenue Molière, 67098 Strasbourg Cedex, France. ${ }^{2}$ Fédération de Médecine Translationnelle de Strasbourg (FMTS), Université de Strasbourg, Strasbourg, France. ${ }^{3}$ Institut de Génétique et de Biologie Moléculaire et Cellulaire (IGBMC), INSERM-U964/CNRS-UMR7104/Université de Strasbourg, 67404 IIIkirch, France. ${ }^{4}$ Département d'informations médicales, Hôpitaux universitaires de Strasbourg, Hôpital civil, 1 place de l'Hôpital, 67000 Strasbourg, France. ${ }^{5}$ Service de Diagnostic génétique, Nouvel hôpital civil, 67000 Strasbourg Cedex, France.

Received: 10 September 2015 Accepted: 10 November 2016

Published online: 23 November 2016

\section{References}

1. Deuschl G, Bain P, Brin M. Consensus statement of the movement disorder society on tremor. Ad Hoc scientific committee. Mov Disord Off J Mov Disord Soc. 1998:13 Suppl 3:2-23.

2. Jankovic J. Essential tremor: a heterogenous disorder. Mov Disord Off J Mov Disord Soc. 2002;17(4):638-44.

3. Hubble JP, Busenbark KL, Pahwa R, Lyons K, Koller WC. Clinical expression of essential tremor: effects of gender and age. Mov Disord Off J Mov Disord Soc. 1997;12(6):969-72

4. Phibbs F, Fang JY, Cooper MK, Charles DP, Davis TL, Hedera P. Prevalence of unilateral tremor in autosomal dominant essential tremor. Mov Disord Off J Mov Disord Soc. 2009;24(1):108-11.
5. Chandran V, Pal PK. Essential tremor: beyond the motor features. Parkinsonism Relat Disord. 2012;18(5):407-13.

6. Louis ED. Essential tremor: evolving clinicopathological concepts in an era of intensive post-mortem enquiry. Lancet Neurol. 2010;9(6):613-22.

7. Kuhlenbäumer G, Hopfner F, Deuschl G. Genetics of essential tremor: meta-analysis and review. Neurology. 2014;82(11):1000-7.

8. Merner ND, Girard SL, Catoire H, Bourassa CV, Belzil W, Rivière J-B, et al. Exome sequencing identifies FUS mutations as a cause of essential tremor. Am J Hum Genet. 2012:91(2):313-9.

9. Jiménez-Jiménez FJ, García-Martín E, Lorenzo-Betancor O, Pastor P, Alonso-Navarro H, Agúndez JAG. LINGO1 and risk for essential tremor: results of a meta-analysis of rs9652490 and rs1 1856808. J Neurol Sci. 2012;317(1-2):52-7.

10. Thier S, Lorenz D, Nothnagel M, Poremba C, Papengut F, Appenzeller S, et al. Polymorphisms in the glial glutamate transporter SLC1A2 are associated with essential tremor. Neurology. 2012;79(3):243-8.

11. Yu S, Chen C-M, Chen Y-C, Chang CW, Chang H-S, Lyu R-K, et al. SLC1A2 variant is associated with essential tremor in Taiwanese population. PLoS ONE. 2013;8(8):e71919.

12. Unal Gulsuner $\mathrm{H}$, Gulsuner $\mathrm{S}$, Mercan $\mathrm{FN}$, Onat $\mathrm{OE}$, Walsh $\mathrm{T}$, Shahin $\mathrm{H}$, et al. Mitochondrial serine protease HTRA2 p.G399S in a kindred with essential tremor and Parkinson disease. Proc Natl Acad Sci U S A. 2014;111(51):18285-90.

13. Stamelou M, Charlesworth G, Cordivari C, Schneider SA, Kägi G, Sheerin U-M, et al. The phenotypic spectrum of DYT24 due to ANO3 mutations. Mov Disord Off J Mov Disord Soc. 2014;29(7):928-34.

14. Elble R, Comella C, Fahn S, Hallett M, Jankovic J, Juncos JL, et al. Reliability of a new scale for essential tremor. Mov Disord Off J Mov Disord Soc. 2012;27(12):1567-9

15. Apartis E, Blancher A, Meissner WG, Guyant-Maréchal L, Maltête D, De Broucker T, et al. FXTAS: new insights and the need for revised diagnostic criteria. Neurology. 2012;79(18):1898-907.

16. Whaley NR, Putzke JD, Baba Y, Wszolek ZK, Uitti RJ. Essential tremor: phenotypic expression in a clinical cohort. Parkinsonism Relat Disord. 2007:13(6):333-9.

17. Hopfner F, Stevanin G, Müller SH, Mundwiller E, Bungeroth M, Durr A, et al. The impact of rare variants in FUS in essential tremor. Mov Disord Off J Mov Disord Soc. 2015;30(5):721-4.

18. Lou JS, Jankovic J. Essential tremor: clinical correlates in 350 patients. Neurology. 1991;41(2 (Pt 1)):234-8.

19. Hopfner F, Erhart T, Knudsen K, Lorenz D, Schneider SA, Zeuner KE, et al. Testing for alcohol sensitivity of tremor amplitude in a large cohort with essential tremor. Parkinsonism Relat Disord. 2015;21:848-51.

20. Elble RJ. Essential tremor frequency decreases with time. Neurology. 2000;55(10):1547-51

21. Chuang W-L, Lu C-S, Huang Y-Z, Chen R-S. Clinical characteristics of essential tremor in Taiwan: an exploratory-comparative study. Eur I Neurol Off J Eur Fed Neurol Soc. 2012;19(1):135-41.

22. Cohen O, Pullman S, Jurewicz E, Watner D, Louis ED. Rest tremor in patients with essential tremor: prevalence, clinical correlates, and electrophysiologic characteristics. Arch Neurol mars. 2003;60(3):405-10.

23. Minen MT, Louis ED. Emergence of Parkinson's disease in essential tremor: a study of the clinical correlates in 53 patients. Mov Disord Off J Mov Disord Soc. 2008;23(11):1602-5

24. Hopfner F, Müller SH, Lorenz D, Appenzeller S, Klebe S, Deuschl G, et al. Mutations in HTRA2 are not a common cause of familial classic ET. Mov Disord Off J Mov Disord Soc. 2015;30(8):1149-50.

25. Tzoulis C, Zayats T, Knappskog PM, Müller B, Larsen JP, Tysnes O-B, et al. HTRA2 p.G399S in Parkinson disease, essential tremor, and tremulous cervical dystonia. Proc Natl Acad Sci U S A. 2015;112(18):E2268.

26. Deuschl G, Raethjen J, Hellriegel H, Elble R. Treatment of patients with essential tremor. Lancet Neurol. 2011;10(2):148-61. 